\title{
The object model for cyber-physical system's in state space (on the example of the pavement compaction process)
}

\author{
Andrey Prokopev \\ School of Engineering and \\ Construction \\ Siberian Federal University \\ Krasnoyarsk, Russia \\ prok1@yandex.ru
}

\author{
Zhasurbek Nabizhanov \\ School of Space and Information \\ Technology \\ Siberian Federal University \\ Krasnoyarsk, Russia \\ jasur150691@yandex.ru \\ Rurik Emelyanov \\ School of Engineering and \\ Construction \\ Siberian Federal University \\ Krasnoyarsk, Russia \\ ert-44@yandex.ru
}

\author{
Vladimir Ivanchura \\ School of Space and Information \\ Technology \\ Siberian Federal University \\ Krasnoyarsk, Russia \\ ivan43ura@yandex.ru
}

\begin{abstract}
We has considered results of object model theoretical description of the cyber-physical system - the compaction process of the road surface with a vibration roller, based on the method of the state space. The working body of the roller includes a vibration drum with a built-in unbalance shaft. The mathematical model of the process of interaction of the object with the compacted road-building material takes into account the mass of the main structural elements of the vibration roller and the mass of the compacted road material. To describe the characteristics of the compacted material, a rheological model of the viscoelastic Kelvin-Voight body is used. The suitability of the obtained mathematical model based on the results of simulation of the system in the MATLAB/Simulink program is experimentally confirmed
\end{abstract}

Keywords-cyber-physical system, mathematical model, vibration roller, roller, compaction process, road-building mixture, rheological model, state space

\section{INTRODUCTION}

Due to information technology cyber-physical system advances predetermines extension to the most fields, which are based on conventional Internet border of people, things and services [1]. CPS technique is Internet of Things (IoT) [2]. Priority tasks of road sector digitizing are designing of cyber-physical system for road construction management, intelligent construction, intelligent compaction [3, 4], continuous compaction control, CCC $[5,6]$, intelligent neural network system of automatic control of vibration roller [7, 8], driverless vibration roller $[9,10]$, automated road building package "paver - vibration roller" [11]. Updating of physical objects such as pavers, vibration rollers as cyber-physical systems elements is the relevant scientific field [12-15].

To implement the concept of a digital economy in the road-building industry, taking into account world achievements, it is necessary to solve the scientific problem of creating domestic systems for continuous quality monitoring of road pavement, which is of major socioeconomic importance. A significant disadvantage of overseas CCC systems is their high cost, comparable to the price of a new domestic vibration roller. The task of the Russian specialists is to propose a technical solution for the system of continuous non-destructive testing of compaction for asphalt pavers and vibratory rollers, which has a low cost and ease of operation. Cybernetic physics studies physical systems by cybernetic methods [16]. Designing a physical object as an element of CPS begins with the choice of an object model and a control goal. Efficiency of CPS designing relies upon level of adequacy of object's mathematical model.

The work is devoted to the theoretical description of the object of the cyber-physical system - a model of interaction between the vibration roller and the medium to be sealed. Compacted asphalt pavement is the result of the implementation of the vibratory roller workflow. Theoretical studies of soil and asphalt mixes compaction are reviewed and carried out by many Russian [17, etc.] and foreign scientists [18-23, etc.]. The disadvantages of the previously developed mathematical models of the process under study are computational difficulties in the design of control systems, the study of dynamic systems to which the object in question belongs. To eliminate these drawbacks and improve the efficiency of theoretical studies of the CPS object using the modern MATLAB/Simulink program, it is recommended to use the state space method, which allows formalization and automation of computational procedures [24]. Matrix notation used in the state space method has an advantage in numerical solution, and the clarity of mathematical formulations and solutions themselves do not fade even for multidimensional MIMO systems (Multiple Inputs Multiple Outputs Systems) describing the behavior of complex production complexes [25].

The aim of the work is a mathematical model designing of the compaction process of a road surface by a vibration roller based on the state space method.

The object of study is the process of compacting the road surfaces with a vibration roller. 
The subject of study is the dependence of the dynamic parameters of the workflow on the operating modes of the vibration roller and the material's characteristics.

\section{MATHEMATICAL FORMULATION OF THE TASK}

The traditional understanding of the process of compacting a material with a drum of a road roller comes down to the usual deformation by stamp, but a curvilinear shape, under the influence of static or dynamic forces with the accumulation of residual deformations for a certain number of repetitive sealing effects.

When a vibrating roller interacts with a medium, depending on the density of the material, the motion can be periodic or chaotic. The normal condition of the compaction process is the periodic movement of the drum, which can be with constant contact, periodic separation, double "jump", swaying [5]. Rational condition for the compaction process is the periodic movement of the drum with constant contact.

Frequency, amplitude, speed and acceleration are the main parameters of oscillations of the drum and particles of the material being compacted. The amplitude of oscillations of any seal depends on the physical and mechanical properties of the material and changes in the process of its compaction. Therefore, the proposed values of the amplitudes of oscillations in the technical characteristics of compactors should be adjusted to the rheological properties of the material [21].

In compiling a mathematical model, the following assumptions are made:

1. machine design elements have absolute rigidity;

2. vibrating working body operates in the unstressed mode with harmonic oscillations;

3. compacted layer has elastic-viscous properties;

4. only the vertical component of vibration is considered;

5. the inertial properties of the medium being sealed are taken into account.

To simplify the study of the object, the design of a twodrum asphalt vibration roller is presented as a combination of two parts - the front and rear. The computational diagram of a three-mass dynamic model of the interaction of the front part of a vibration roller with a material during compaction is shown in Fig. 1. The Kelvin-Voigt body is use for the theoretical description of the material being compacted.

In the diagram of fig. 1 the following notation is used: $m_{d}$ - drum weight, $\mathrm{kg} ; m_{f}$ - frame weight, $\mathrm{kg} ; m_{s}$ - the mass of material to be compacted under the roller, participating in the vibration system, $\mathrm{kg} ; k_{d f}$ - elastic coefficient of shock absorbers, newton meter, $\mathrm{N} / \mathrm{m} ; c_{d f}-$ shock absorber damping ratio, $\mathrm{Ns} / \mathrm{m} ; k_{s}$ - elastic coefficient of the material under the roller, $\mathrm{N} / \mathrm{m} ; c_{s}$ - material's damping ratio under the roller, Ns/m; $y_{s}, y_{d}, y_{f}$ - movement of elements of a dynamic system, $\mathrm{m}$.

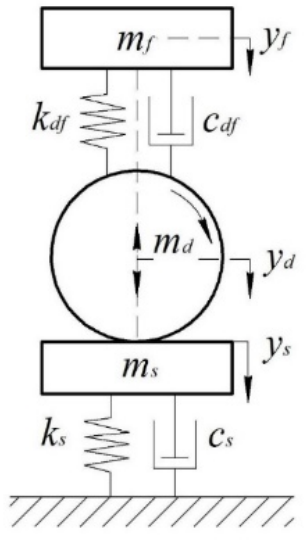

Fig. 1. Dynamic model of the system "roller frame - vibratory drum - material"

As a result of analyzing the mixture compaction process by a vibration roller (Fig. 1), its mathematical model was compiled, which simultaneously reflects both the vibration dynamics of the vibration rollers and the rheological properties of the material being compacted. We deduced differential equations of the oscillatory system "frame vibrating drum - material" in the form of a system

$$
\begin{aligned}
& \left(m_{d}+m_{s}\right) \cdot \ddot{y}_{d}+c_{s} \cdot \dot{y}_{d}+c_{d f} \cdot\left(\dot{y}_{d}-\dot{y}_{f}\right) \\
& +k_{s} \cdot y_{d}+k_{d f} \cdot\left(y_{d}-y_{f}\right) \\
& =\left(m_{d}+m_{s}\right) \cdot g+F_{e} \cdot \sin (\omega \cdot t) \text {; } \\
& m_{f} \cdot \ddot{y}_{f}-c_{d f} \cdot\left(\dot{y}_{d}-\dot{y}_{f}\right)-k_{d f} \cdot\left(y_{d}-y_{f}\right)=m_{f} \cdot g \text {; } \\
& F_{e}=m_{e} \cdot r_{e} \cdot \omega^{2} ; \dot{y}_{s}=\dot{y}_{d} ; y_{s}=y_{d} \text {; } \\
& \text { by } t=0, \dot{y}_{d}=\dot{y}_{s}=0 ; y_{d}=y_{s}=0 \text {, }
\end{aligned}
$$

where $y_{d}, \dot{y}_{d}, \ddot{y}_{d}$ - displacement, speed, acceleration of the vibration roller, respectively; $g$ - gravitational acceleration, $\mathrm{m} / \mathrm{s}^{2} ; F_{e}$ - disturbing force of the vibration exciter, $\mathrm{N} ; t-$ time, $\mathrm{s} ; m_{e}-$ unbalance mass of vibrator, $\mathrm{kg} ; r_{e}$ - vibrator unbalance eccentricity radius, $m$; $\omega$ - angular frequency of revolution of vibration exciter shaft, $\mathrm{rad} / \mathrm{s}$.

Taking into account the accepted notation, we define the contact force arising from the interaction of the vibration roller and the medium to be sealed

$$
\begin{aligned}
F_{c} & =m_{s} \cdot \ddot{y}_{1}-m_{s} \cdot g+k_{s} \cdot y_{d}+c_{s} \cdot \dot{y}_{d} \\
& =m_{e} \cdot r_{e} \cdot \omega^{2} \cdot \sin (\omega \cdot t)+m_{d} \cdot g \\
& +m_{f} \cdot g-m_{d} \cdot \ddot{y}_{d}-m_{f} \cdot \ddot{y}_{f} .
\end{aligned}
$$

Based on the system of equations (1), you can determine the acceleration of the roller 
$\ddot{y}_{d}=\frac{\left[\begin{array}{c}-c_{s} \cdot \dot{y}_{d}-c_{d f} \cdot\left(\dot{y}_{d}-\dot{y}_{f}\right) \\ -k_{s} \cdot y_{d} \\ -k_{d f} \cdot\left(y_{d}-y_{f}\right) \\ +F_{e} \cdot \sin (\omega \cdot t)\end{array}\right]}{m_{d}+m_{s}}+g$.

III. MATHEMATICAL MODEL IN THE STATE SPACE

The state space method allows us to represent the control system (1) in the following form [24, 26]:

$$
\begin{aligned}
& \dot{x}(t)=A(t) \cdot x(t)+B(t) \cdot u(t) ; \\
& y(t)=C(t) \cdot x(t)+D(t) \cdot u(t),
\end{aligned}
$$

where $x(t)$-dimension state vector $(n \times 1)$, the components of which are state variables of the $n$-th order system, $x(t)=\left[x_{1}(t), x_{2}(t), \ldots, x_{n}(t)\right]^{T} ; y(t)-$ dimension output vector $(p \times 1)$, whose components are output variables of the system, $y(t)=\left[y_{1}(t), y_{2}(t), \ldots, y_{p}(t)\right]^{T} ; u(t)$ - dimension input vector $(r \times 1)$, whose components are input variables of the system, $u(t)=\left[u_{1}(t), u_{2}(t), \ldots, u_{r}(t)\right]^{T} ; A(t)-$ system coefficient matrix $(n \times n) ; B(t)-$ input matrix $(n \times m)$; $C(t)$ - output matrix $(p \times n), p$ - number of output values; $D(t)$ - detour matrix $(p \times m)$, which determines the direct dependence of the output on the input.

For further transformations, the system of equations (1) is reduced to the following form:

$$
\ddot{y}_{d}=\frac{\left[\begin{array}{c}
-k_{d f} \cdot\left(y_{d}-y_{f}\right)-c_{d f} \cdot\left(\dot{y}_{d}-\dot{y}_{f}\right) \\
-k_{s} \cdot y_{d}-c_{s} \cdot \dot{y}_{d} \\
+\left(m_{d}+m_{s}\right) \cdot g \\
+F_{e} \cdot \sin (\omega \cdot t)
\end{array}\right]}{\left(m_{d}+m_{s}\right)} ;
$$

$\ddot{y}_{f}=\frac{1}{m_{f}}\left[\begin{array}{c}k_{d f} \cdot\left(y_{d}-y_{f}\right) \\ +c_{d f} \cdot\left(\dot{y}_{d}-\dot{y}_{f}\right) \\ +m_{f} \cdot g\end{array}\right]$;

$$
F_{e}=m_{e} \cdot r_{e} \cdot \omega^{2} \text {. }
$$

Initial data:

at $t=0, \dot{y}_{1}=\dot{y}_{s}=0 ; y_{1}=y_{s}=0$.

System state variables are defined: $z_{1}$ - vertical movement of the drum, $z_{1}=y_{d} ; z_{2}$ - vertical speed of the drum, $z_{2}=\dot{y}_{d} ; z_{3}$ - vertical movement of frame, $z_{3}=y_{f}$; $z_{4}$ - speed of vertical frame's movement, $z_{3}=\dot{y}_{f}$.
The system of equations (5) after transformations in the Cauchy normal form:

$\dot{z}_{1}=z_{2}$

$\dot{z}_{2}=\frac{\left[\begin{array}{c}-k_{d f} \cdot\left(z_{3}-z_{1}\right) \\ -c_{d f} \cdot\left(z_{4}-z_{2}\right) \\ -k_{s} \cdot z_{3}-c_{s} \cdot z_{4} \\ +F_{e} \cdot \sin (\omega \cdot t)\end{array}\right]}{\left(m_{d}+m_{s}\right)}+g ;$

$\dot{z}_{3}=z_{4}$;

$\dot{z}_{4}=\frac{1}{m_{f}}\left[\begin{array}{c}k_{d f} \cdot\left(z_{3}-z_{1}\right) \\ +c_{d f} \cdot\left(z_{4}-z_{2}\right)\end{array}\right]+g$.

Model of the control object in the state space, in vectormatrix form

$$
\begin{aligned}
& A=\left[\begin{array}{cccc}
0 & 1 & 0 & 0 \\
-\frac{k_{s}+k_{d f}}{T} & -\frac{c_{s}+c_{d f}}{T} & \frac{k_{d f}}{T} & \frac{c_{d f}}{T} \\
0 & 0 & 0 & 1 \\
\frac{k_{d f}}{m_{f}} & \frac{c_{d f}}{m_{f}} & -\frac{k_{d f}}{m_{f}} & -\frac{c_{d f}}{m_{f}}
\end{array}\right] \\
& B=\left[\begin{array}{cc}
0 & 0 \\
\frac{1}{m_{d}+m_{s}} & 0 \\
0 & 0 \\
0 & \frac{1}{m_{f}}
\end{array}\right] \\
& C=\left[\begin{array}{llll}
1 & 0 & 0 & 0 \\
0 & 1 & 0 & 0
\end{array}\right] ; D=\left[\begin{array}{ll}
0 & 0 \\
0 & 0
\end{array}\right] \text {, }
\end{aligned}
$$

where $T=m_{d}+m_{s}$; input vector

$$
u(t)=\left[\begin{array}{c}
m_{e} \cdot r_{e} \cdot \omega^{2} \cdot \sin (\omega \cdot t)+m_{d} \cdot g+m_{s} \cdot g \\
m_{f} \cdot g
\end{array}\right] .
$$

The model of the control object is obtained by the state space method.

\section{STUDY OF THE MATHEMATICAL MODEL}

The state space method allows us to represent the control system (1) in the following form [24, 26]:

To assess the adequacy of the mathematical model of the process, calculations were performed in the MATLAB/Simulink program environment. A simulation 
model of the process under investigation is presented in Fig. 2.

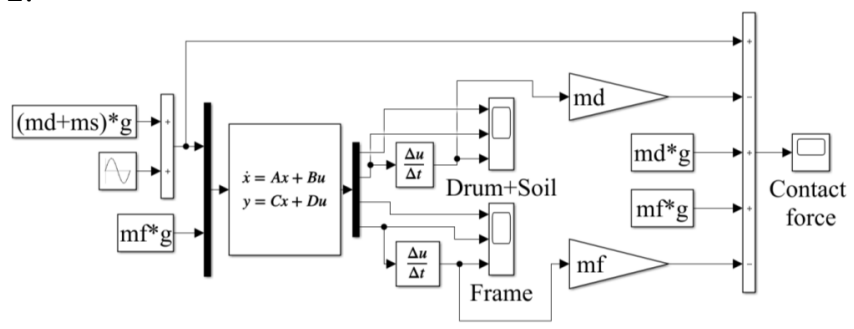

Fig. 2. Simulation model of the control object in the MATLAB/Simulink language

To simulate the process, the source data from the work [27] was used. Source data:

$$
\begin{aligned}
& k_{d f}=3.4 \cdot 10^{6} \mathrm{~N} / \mathrm{m} ; k_{s}=58 \cdot 10^{6} \mathrm{~N} / \mathrm{m} ; \\
& c_{s}=180 \cdot 10^{3} \mathrm{~N} \cdot \mathrm{s} / \mathrm{m} ; \mathrm{c}_{d f}=k_{d f} \cdot \eta / \omega ; \\
& \eta=0.16 ; m_{f}=4565 \mathrm{~kg} ; m_{d}=2638 \mathrm{~kg} ; \\
& m_{s}=0.2 \cdot m_{d}, \mathrm{~kg} ; m_{e} \cdot r_{e}=2.32 \mathrm{~kg} \cdot \mathrm{m} ; \\
& \omega=2 \cdot \pi \cdot f, \mathrm{rad} / \mathrm{s} ; f=27 \mathrm{~Hz} .
\end{aligned}
$$

The source data is implemented in the $m$-file in the language of the program MATLAB. As a result of computer simulation, the time dependences of the parameters of the drum's vibration process and the roller frame during compaction of the road building mixture were obtained: movement; speed; acceleration.

Figure 3 shows the time dependences of the acceleration of the roller and the frame when modeling the workflow.

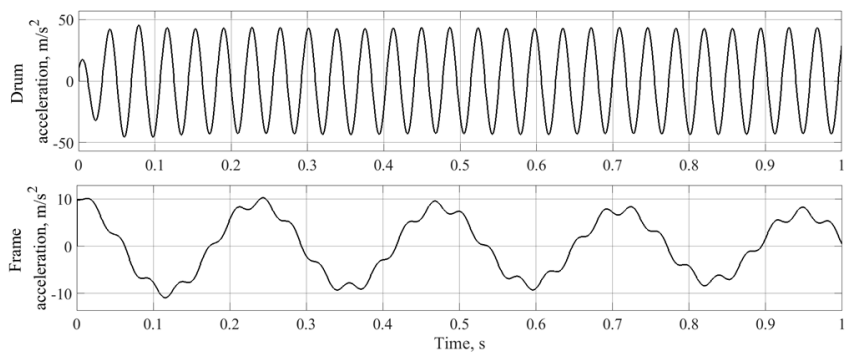

Fig. 3. The dependencies of the acceleration of the drum and the frame of the vibration roller

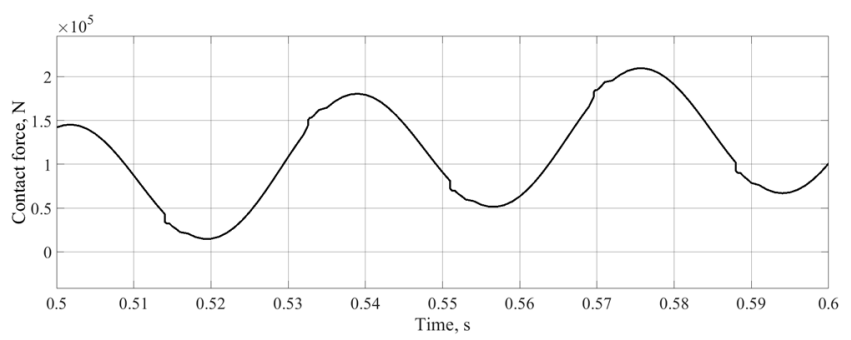

Fig. 4. The dependence of the contact drum of the roller on the material to be compacted

The adequacy of the developed mathematical and simulation models of the study object is confirmed by simulation modeling.
Graphic dependences of vertical acceleration are received, fig. 3, drums and frames, the values of which have a good agreement with the data of the experimental work [27]. Harmonic dependencies are linear. This corresponds to the initial and middle stages of compaction of the pavement by vibration rollers.

Dependence of the contact force of the roller, fig. 4, during the compaction of the material is characterized as harmonic with signs of nonlinearity. It is linked with parameters of the compaction process, the vibration roller and the material. The values of the contact force adequately correspond to the data obtained by the authors in a scientific work [27].

\section{THE DEFINITION OF THE TRANSFER FUNCTION OF THE CONTROL OBJECT}

The task of determining the transfer function (PF) was performed in order to conduct a study of a mathematical model using block modeling software. Using the functions of the MATLAB/Simulink program, the transfer functions of the control object for the output signals of the motion and velocity of the roller's vibrating roller are obtained:

As a result of the implementation of the MATLAB functions, the input transfer functions with an adjustable variable - the angular frequency of vibration - were obtained:

PF of controlled value - drum displacement

$$
W_{d}(s)=\frac{\left[\begin{array}{c}
0,0003159 \cdot s^{2}+0,0002219 \cdot s \\
+0,2353
\end{array}\right]}{\left[\begin{array}{c}
s^{4}+58,58 \cdot s^{3}+2,018 \cdot 10^{4} \cdot s^{2} \\
+5,522 \cdot 10^{4} \cdot s+1,365 \cdot 10^{7}
\end{array}\right]}
$$

PF of controlled value - drum velocity

$$
W_{v}(s)=\frac{\left[\begin{array}{c}
0,0003159 \cdot s^{3}+0,0002219 \cdot s^{2} \\
+0,2353 \cdot s
\end{array}\right]}{\left[\begin{array}{c}
s^{4}+58,58 \cdot s^{3}+2,018 \cdot 10^{4} \cdot s^{2} \\
+5,522 \cdot 10^{4} \cdot s+1,365 \cdot 10^{7}
\end{array}\right]} .
$$

Transfer functions are correct format. The simulation results showed good reproducibility of the compaction pavement workflow with a vibration roller.

\section{CONCLUSIONS}

We obtained a mathematical model of the object of the cyber-physical system in the state space - the process of compaction of the road surface by a vibration roller. An experimental test of its adequacy has been performed. The models of transfer functions for the output signals of displacement and velocity of the vibration roller, the roller frame are determined. The transfer functions have the fourth order, which complicates the task of designing the parameters of the automatic control system's controller.

The results of the work are a stage of research work in the field of cyber-physical system design for road construction. 


\section{REFERENCES}

[1] R. Anderl, M. Eigner, U. Sendler, R. Stark, Smart Engineering Interdisziplinäre Produktentstehung. Acatech Diskussion. Springer, 2012, $58 \mathrm{p}$

[2] D. Serpanos, M. Wolf, Internet-of-Things (IoT) Systems Architectures, Algorithms, Methodologies. Springer, Cham, 2018, 95 p.

[3] Q. Xu, G.K. Chang, "Adaptive quality control and acceptance of pavement material density for intelligent road construction". Automation in Construction 62, 2015, pp. 78-88.

[4] G.K. Chang, K. Mohanraj, W.A. Stone, D.J. Oesch, V. (Lee) Gallivan, "Leveraging Intelligent Compaction and Thermal Profiling Technologies to Improve Asphalt Pavement Construction Quality: A Case Study". Transportation Research Record Journal of the Transportation Research Board. 2672(26), 2018, pp. 48-56.

[5] J. Pistrol, S. Villwock, W. Völkel, F. Kopf, D. Adam, "Continuous Compaction Control (CCC) with Oscillating Rollers". Procedia Engineering. 143, 2016, pp. 514-521.

[6] W. Hu, X. Shu, B. Huang, M. Woods, "Field investigation of intelligent compaction for hot mix asphalt resurfacing". Frontiers of Structural and Civil Engineering. 11(1), 2017, pp. 47-55.

[7] M. Barman, M. Nazari, S.A. Imran, S. Commuri, M. Zaman, F. Beainy, D. Singh, "Quality Control of Subgrade Soil Using Intelligent Compaction". Innovative Infrastructure Solutions. 1(1), 2016, p. 23.

[8] M. Barman, S.A. Imran, M. Nazari, S. Commuri, M. Zaman, "Use of Intelligent Compaction in Detecting and Remediating UnderCompacted Spots During Compaction of Asphalt Layers". In: Hossain Z., Zhang J., Chen C. (eds) Solving Pavement and Construction Materials Problems with Innovative and Cutting-edge Technologies. GeoChina 2018. Sustainable Civil Infrastructures. Springer, Cham, 2019, pp. 131-141.

[9] X. Fang, Y. Bian, M. Yang, G. Liu, "Development of a path following control model for an unmanned vibratory roller in vibration compaction". Advances in Mechanical Engineering. 10(5), 2018, pp. $1-16$.

[10] Y. Bian, X. Fang, M. Yang, Z. Zhao, “Automatic Rolling Control for Unmanned Vibratory Roller Based on Fuzzy Algorithm”. Journal of Tongji University (Natural Science). 45(12), 2017, pp. 1831-1838.

[11] S. Zhu, X. Li, Wang, D. Yu, "Development of an Automated Remote Asphalt Paving Quality Control System". Transportation Research Record. 2672(26), 2018, pp. 28-39.

[12] D.H. Liu, Z.L. Li, Z.H. Lian, "Compaction quality assessment of earthrock dam materials using roller integrated compaction monitoring technology". Automat. Constr. 44, 2014, pp. 234-246.
[13] B. Kenneally, O.M. Musimbi, J. Wang, "Finite element analysis of vibratory roller response on layered soil systems". Comput Geotech. 67, 2015, pp. 73-82.

[14] J. Li, Z. Zhang, H. Xu, "Dynamic characteristics of the vibratory roller test-bed vibration isolation system: simulation and experiment". J Terramechanics. 56, 2014, pp. 139-156.

[15] Xu, Q., Chang, G.K.: “Adaptive quality control and acceptance of pavement material density for intelligent road construction". Automat. Constr. 62, 2016, pp. 78-88.

[16] P. Kyung-Joon, R. Zheng, X. Liu, "Cyber-physical systems: Milestones and research challenges". Computer Communications. 36, 2012, pp. 1-7.

[17] V.V. Mikheyev, S.V. Saveliev, "Modelling of deformation process for the layer of elastoviscoplastic media under surface action of periodic force of arbitrary type”. J. Phys.: Conf. Ser. 944(1), 2018, 012079.

[18] R.V. Rinehart, "Instrumentation of a roller compactor to monitor vibration behavior during earthwork compaction". J. Automation in Construction. 17(2), 2008, pp. 144-150.

[19] D. White, M. Thompson, "Relationships Between In Situ and RollerIntegrated Compaction Measurements for Granular Soils". Journal of Geotechnical and Geoenvironmental Engineering. 134(12), 2008, pp. 1763-1770

[20] S. Bejan, "The Roller-Ground Dynamic Interaction in The Compaction Process Through Vibrations for Road Construction". Romanian Journal of Transport Infrastructure.5(2), 2016, pp. 1-9.

[21] F. Beainy, S. Commuri, M. Zaman, "Dynamical response of vibratory rollers during the compaction of asphalt pavements". J. Eng. Mech. 140(7), 2014, 04014039.

[22] S.A. Imran, S. Commuri, M. Barman, M. Zaman, F. Beainy, "Modeling the dynamics of asphalt-roller interaction during compaction". Journal of Construction Engineering and Management. 143(7), 2017, pp. 1763-1770.

[23] Sh. Li, Ch. Hu, "Study on Dynamic Model of Vibratory Roller - Soil System". IOP Conf. Series: Earth and Environmental Science 113, 2018, 012187.

[24] P.M. Derusso, R.J. Roy, Ch.M. Close, State variables for engineers. New York; London: John Wiley \& Sons, 1965, 608 p.

[25] V. Strejc, State Space Theory of Discrete Linear Control. John Wiley \& Sons, 1981, 426 p.

[26] Ch.L. Phillips, R.D. Harbor, Feedback Control Systems. Pearson, 2010, $784 \mathrm{p}$

[27] Paul J. van Susante, Michael A. Mooney, "Capturing Nonlinear Vibratory Roller Compactor Behavior through Lumped Parameter Modeling". Journal of Engineering Mechanics. 134(8), August 2008. 